\title{
APLICACIÓN BÁSICA DE VISIÓN ARTIFICIAL PARA DETERMINAR EL ÁREA DE UNA PIEZA DE CUERO VACUNO
}

\author{
Pedro HUAMANÍ NAVARRETE \\ Facultad de Ingeniería \\ phuamani@urp.edu.pe
}

\begin{abstract}
RESUMEN
Este artículo presenta una aplicación básica de la visión artificial para determinar el área de una pieza de cuero de un ganado vacuno. Para ello, se emplearon técnicas matemáticas de procesamiento digital de imágenes basadas en la manipulación del histograma a través de una potencia, seguido de una detección de múltiple umbral haciendo uso del método de Otsu, para posteriormente binarizar la imagen digital y aplicar los operadores morfológicos de erosión y cerradura que permitieron limpiar el ruido del contorno, así como facilitar la cuenta del número de pixeles blancos contenidos en el área de interés. Las técnicas señaladas fueron desarrolladas algorítmicamente en el software Matlab con el complemento del Toolbox Image Processing. En cuanto a las fotografías de las piezas de cuero, se utilizaron 10; algunas de ellas presentaron resoluciones distintas, pero todas con igual codificación de bits por pixel. Estas imágenes fueron obtenidas directamente de diferentes sitios de internet, tratando en lo posible de representar a las diferentes razas de ganado vacuno existentes. Por otro lado, para calcular el área, se tuvo que suponer un dato importante que viene hacer el tamaño del pixel en $\mathrm{cm} 2$ tomado con ayuda del trabajo de L. Sánchez y otros [07]. De esta forma, el área obtenida para las 10 imágenes de piezas de cuero fluctuó entre 38.7478 y 48.0689 pies2, lo que significó una concordancia respecto a una verificación visual sobre cada una de las imágenes analizadas.
\end{abstract}

\section{PALABRAS CLAVE}

Piezas de cuero vacuno, manipulación del histograma, método de Otsu, erosión y cerradura.

\section{BASIC APPLICATION OF ARTIFICIAL VISION TO DETERMINE THE AREA OF ONE PIECE LEATHER VACCINE}

\section{SUMMARY}

This article presents a basic application of artificial vision to determine the area of a piece of leather of a cattle. For this, mathematical techniques of digital image processing based on the manipulation of the histogram through a power were employed, followed by a detection of multiple threshold using the Otsu method, 
to later binarize the digital image and apply the morphological operators of erosion and lock that allowed to clean the contour noise, as well as to facilitate the count of the number of white pixels contained in the area of interest. The indicated techniques were developed algorithmically in the Matlab software with the Toolbox Image Processing add-on. As for the photographs of the leather pieces, 10 were used; some of them presented different resolutions, but all with the same coding of bits per pixel. These images were obtained directly from different internet sites, trying as much as possible to represent the different breeds of cattle. On the other hand, to calculate the area, it was necessary to suppose an important fact that the pixel size in $\mathrm{cm} 2$ has been taken with the help of the work of L. Sánchez et al. [07]. In this way, the area obtained for the 10 images of leather pieces fluctuated between 38.7478 and $48.0689 \mathrm{ft} 2$, which meant a concordance with respect to a visual verification of each of the images analyzed.

\section{KEYWORDS}

Cow leather pieces, histogram manipulation, Otsu method, erosion and lock.

Recibido: $14 / 8 / 2017$

Aprobado: $30 / 11 / 2017$ 


\section{INTRODUCCIÓN}

En nuestros días, la demanda por adquirir prendas y artículos fabricados en cuero se ha incrementado notablemente. Por lo cual, el comercio de piezas o planchas de cuero de animales se vuelve más frecuente; particularmente, el cuero del ganado vacuno resulta ser el más empleado por caracterizarse como el más rígido, flexible, resistente y por poseer una suavidad al tacto.

Por tal razón, en nuestro país son muchos los empresarios que han apostado por implementar curtiembres, con la finalidad de convertir la materia orgánica de un animal (piel), en una inorgánica (pieza o plancha de cuero). Y para ello, se realizan varias etapas como son la limpieza de la piel eliminando el pelo, la grasa y la carne con ayuda de productos químicos. Luego, se procede a curtir la piel utilizando cromo o tanino. Posteriormente, se continúa con el recurtimiento que incluye la tintura. Finalmente, se procede a secar, revestir y lavar la piel del animal, para obtener el producto final que es el cuero .

Sin embargo, tal producto final presenta un contorno amorfo, lo cual dificulta la medición y por lo tanto el área en la unidad de pies elevado al cuadrado termina no siendo la más exacta. Es por ello, que en esta parte final del proceso de preparación de la pieza o plancha de cuero, las pequeñas curtiembres utilizan una plancha de metal para determinar la superficie de la manera más aproximada; pues, utilizar una mesa o máquina medidora, por el precio elevado que tienen, haría más cara la producción de las piezas de cuero y sobre todo cuando la cantidad producida diariamente, es reducida.

Por tal razón, en este artículo se presenta una alternativa de medición del área de una pieza de cuero haciendo uso de la visión artificial, la cual viene hacer un campo de la inteligencia artificial que hace uso de técnicas matemáticas de procesamiento digital de imágenes, para analizar, manipular y obtener cualquier tipo de información desde una imagen digital tal como lo haría una persona cuando hace uso de su sentido de la vista para orientarse.

Por otro lado, según Szeliski [06],"la visión por computadora se encarga de describir el mundo que vemos en una o más imágenes con el fin de reconstruir sus propiedades, como la forma, la iluminación y las distribuciones de color". Y, para que ello ocurra se necesita de algoritmos computacionales que puedan desarrollar las diferentes tareas matemáticas, a partir de la captura de una imagen digital haciendo uso de cualquier dispositivo que lo permita.

De esta manera, para desarrollar la aplicación básica de visión artificial que determina el área de una pieza de cuero, se ha optado por utilizar principalmente la manipulación del histograma, la determinación de múltiples umbrales por el método de Otsu, y las transformaciones morfológicas. Si bien es cierto que una operación de detección de contorno, aplicado sobre un objeto de tonalidad oscura en una fotografía de fondo blanco, resulta ser una de las mejores herramientas utilizadas para la etapa de segmentación, también es importante resaltar que no todos los objetos contarán con una 
tonalidad diferente al fondo en donde se localizan. Es así como, en una pieza de cuero en particular, es posible encontrar combinación de tonalidades y colores dependiendo de la raza del ganado vacuno; por lo cual, resulta necesario aplicar herramientas de procesamiento digital de imágenes que logren una adecuada segmentación, para facilitar la cuenta de pixeles en el interior del contorno amorfo que posee una pieza de cuero.

\section{METODOLOGÍA DESARROLLADA}

La metodología utilizada para el desarrollo de este trabajo implicó la participación de tres etapas principales. Como primera etapa, la recopilación de fotografías de piezas de cuero vacuno, las cuales fueron obtenidas del internet y considerando las variadas razas de ganado vacuno existentes. Luego, como segunda etapa, se procedió a aplicar la técnica matemática para detectar en la fotografía el contorno amorfo de la pieza de cuero, y el correspondiente llenado con pixeles de color blanco. Y, como tercera y última etapa, se continuó con la determinación del área a partir de la resolución de la fotografía adquirida y de una medida de distancia referencial. A continuación, se detalla cada una de las etapas desarrolladas.

\section{1) Recopilación de fotografías de piezas de cuero}

Son muchas las ciudades de nuestro territorio que están dedicadas a la agricultura y ganadería, así como también son variadas las razas de ganado vacuno existentes. Y, como es de saber, algunas razas son apropiadas para la industria de la leche y otras para la de carne y cuero. Sin embargo, este artículo se limita a la recopilación de fotografías digitales de piezas de cuero de diversas razas de ganado vacuno. Por lo tanto, se procedió a revisar diversas páginas web del internet para alcanzar un total de 10 imágenes y todas ellas con un fondo de color blanco, pero con resoluciones de pixeles diferentes.

De esta manera, en la figura 01, se muestran 04 fotografías de las piezas de cuero en formato JPG, que fueron leídas y visualizadas con ayuda del comando IMREAD del Toolbox Image Processing del Matlab. A continuación, el código de Matlab empleado para dicha visualización.

$$
\begin{aligned}
& >>11 \text { = imread('cuero 1.jpg'); } \\
& \text { > subplot(221), imshow(11) } \\
& >>12 \text { = imread('cuero2.jpg'); } \\
& \text { > subplot(222), imshow (12) } \\
& >\text { 13 = imread('cuero3.jpg'); } \\
& \text { > subplot(223), imshow( 13) } \\
& >>14 \text { = imread('cuero4.jpg'); } \\
& \text { > subplot(224), imshow( 14) }
\end{aligned}
$$



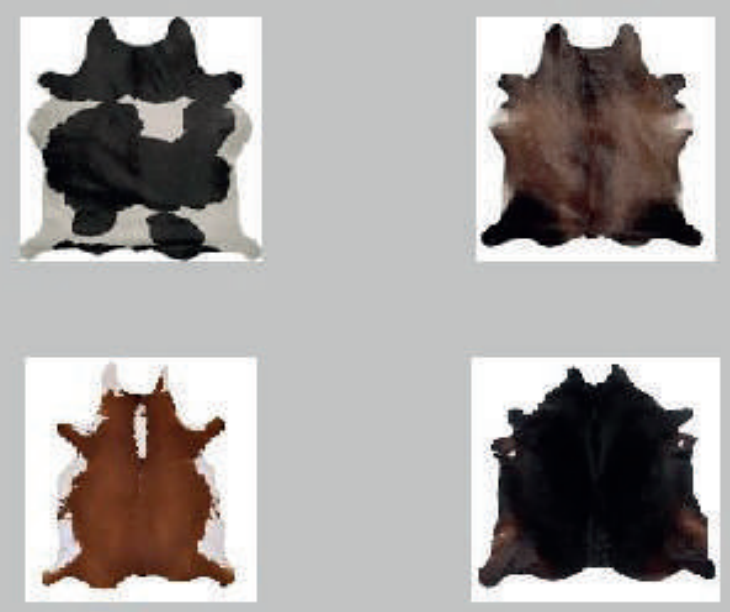

Figura 01. Fotografías de 04 piezas de cuero.

Asimismo, en la figura 02 , se muestran las otras 06 fotografías de las piezas de cuero en formato JPG que fueron empleadas en este artículo.

Tal como se puede apreciar en ambas figuras, algunas de las fotografías presentan una tonalidad oscura prácticamente en su totalidad y con una buena diferenciación del contorno, esto facilitó la segmentación con un umbral elegido a partir del histograma de la imagen analizada y con ello la detección final del borde para la determinación del área. Sin embargo, en otro grupo de fotografías se observa que la pieza de cuero presenta amplias zonas claras (propias de la raza vacuna Holstein) sobre el contorno, lo cual originó limitación al momento de realizar la segmentación directa a partir de un umbral; por ello, se tuvo que recurrir a la manipulación del histograma. Y, un nuevo grupo de imágenes hace correspondencia a una pieza de cuero de color marrón prácticamente en su totalidad, pero con pequeñas manchas de color blanco en el perímetro. Por lo cual, el análisis de contorno en este último grupo de imágenes también resultó limitado, pero solo de manera parcial. 


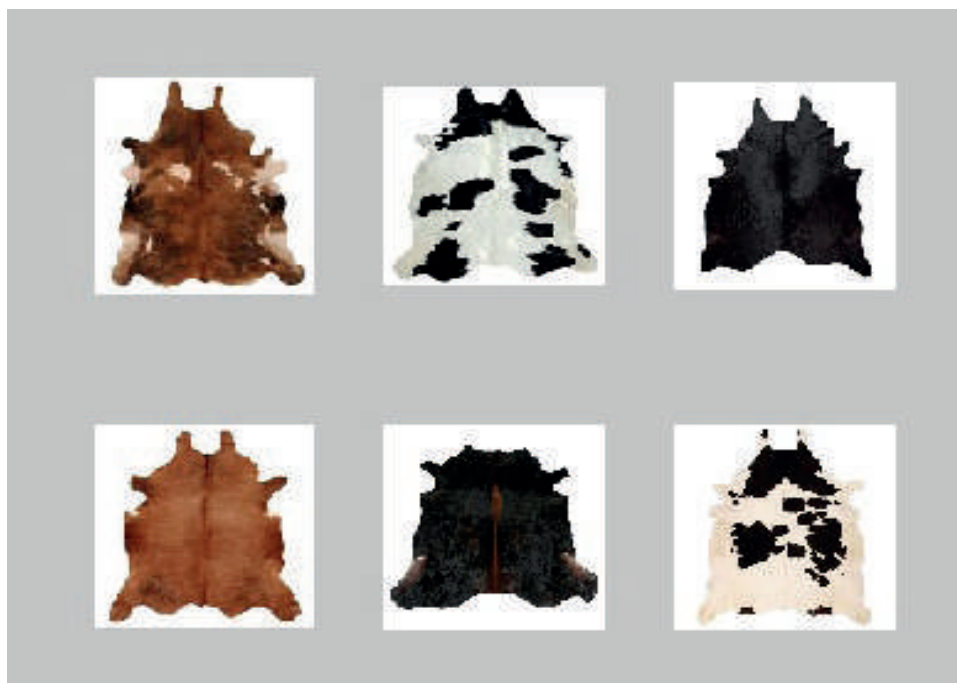

Figura 02. Fotografías de 06 piezas de cuero.

También es importante resaltar, que las fotografías fueron obtenidas de algunos sitios web con ayuda de Google Images, y es por esa razón que presentan resoluciones diferentes, pero con la misma codificación de 24 bits/ pixel (bpp). A continuación, en la tabla 01 , se muestra la resolución de cada una de las fotografías capturadas, y en la sección de bibliografía se presentan los enlaces de donde se obtuvieron.

\section{2) Técnica matemática de detección de contorno}

Tal como fue comentado en la sección anterior, no todas las piezas de cuero presentan un contorno bien definido y diferente en tonalidad con respecto al fondo de la fotografía. Por tal razón, no solamente fue necesario representar el histograma de la imagen digital para obtener un umbral adecuado que permita la binarización, si no también se tuvo que recurrir a la manipulación del histograma complementado con el método de Otsu para lograr la mejor elección de un umbral, lo cual permitió la binarización de la imagen y con ello la segmentación. Posteriormente, con ayuda de las transformaciones morfológicas, se procedió a eliminar el ruido en los contornos y se logró llenar de pixeles blancos el interior del contorno principal de la pieza de cuero.

De esta manera, como primer paso de la técnica matemática de detección de contorno, se realizó una transformación de la imagen de color a imagen con tonos de gris. Tal transformación no fue realizada utilizando la expresión de Baéz y otros [02], porque debido a la naturaleza de la imagen el histograma resultante se concentraba en la tonalidad de gris central. Por lo cual, se procedió de la forma convencional utilizando diferentes pesos para cada uno de los canales de color. Ver la ecuación 01, y luego observar la figura 03 que representa el resultado de la conversión a tonos de gris, con dos fotografías de piezas de cuero. 


\begin{tabular}{|c|c|c|c|}
\hline & Nombre de Archivo & $\begin{array}{c}\text { Resolución (pix- } \\
\text { eles) }\end{array}$ & $\begin{array}{c}\text { Codificación (bits/ } \\
\text { pixel) }\end{array}$ \\
\hline 1 & cuero1.jpg & $457 \times 500$ & $24 \mathrm{bpp}$ \\
\hline 2 & cuero2.jpg & $233 \times 257$ & $24 \mathrm{bpp}$ \\
\hline 3 & cuero3.jpg & $209 \times 241$ & $24 \mathrm{bpp}$ \\
\hline 4 & cuero4.jpg & $217 \times 233$ & $24 \mathrm{bpp}$ \\
\hline 5 & cuero5.jpg & $217 \times 233$ & $24 \mathrm{bpp}$ \\
\hline 6 & cuero6.jpg & $229 \times 220$ & $24 \mathrm{bpp}$ \\
\hline 7 & cuero7.jpg & $225 \times 225$ & $24 \mathrm{bpp}$ \\
\hline 8 & cuero8.jpg & $225 \times 225$ & $24 \mathrm{bpp}$ \\
\hline 9 & cuero9.jpg & $375 \times 375$ & $24 \mathrm{bpp}$ \\
\hline 10 & cuero0.jpg & $325 \times 325$ & $24 \mathrm{bpp}$ \\
\hline
\end{tabular}

Tabla 01. Resolución y codificación de las fotografías de piezas de cuero.

$I_{\text {gris }}=0.299 * \mathrm{I}_{\text {color }_{\text {ROJO }}}+0.587 * \mathrm{I}_{\text {color }_{V E R D E}}+0.114 * \mathrm{I}_{\text {color_AZUL }}$

$>>$ Igris $1=0.299 * 11(:,:, 1)+0.587 * 11(:,:, 2)+0.114 * 11(: ;:, 3)$;

$>>$ Igris2 $=0.299 * 12(: ;, 1)+0.587 * 12(:,:, 2)+0.114 * 12(: ;:, 3)$;

$>>$ subplot(221), imshow( I1), subplot(223), imshow( I2)

> subplot(222), colormap(gray(256)), imshow(Igris1)

> subplot(224), colormap(gray(256)), imshow(Igris2)
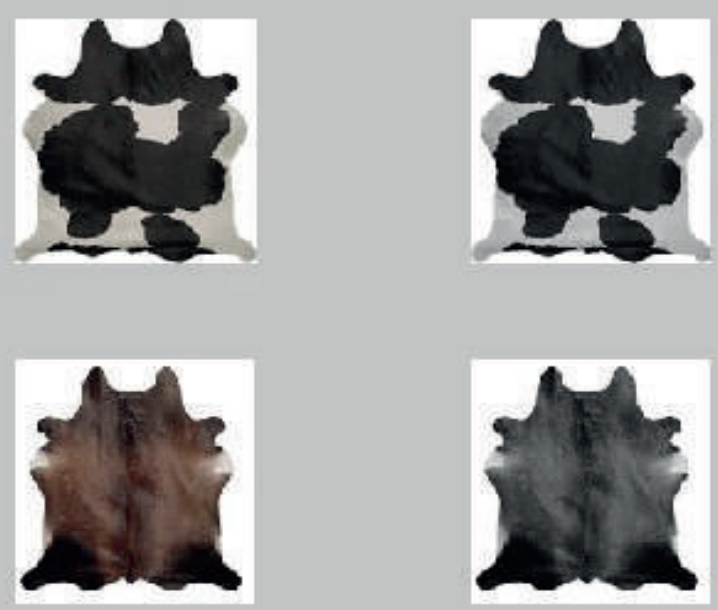

Figura 03. Conversión de imagen de color a tonos de gris. 
Una vez lograda la conversión a gris, se procedió a manipular el histograma basado en una curva de potencia elevado a la cuarta. Ver la ecuación 02 y la figura 04 para observar el efecto de la potencia sobre la imagen de gris definida con la variable "Igris2". Esta operación de potencia sobre los pixeles de la imagen de gris permitió mantener todas las zonas oscuras y asimismo oscurecer aquellas zonas claras, facilitando la visualización bien definida del contorno de la pieza de cuero.

$$
I_{\text {transformada }}=\left\|255 *\left(\frac{\mathrm{I}_{\text {gris }}}{255}\right)^{4}\right\|
$$

El símbolo representa una operación de redondeo dentro del rango dinámico de 0 a 255.

$>>$ Itransformada2 $=\operatorname{uint8}(255 *($ double $($ lgris 2$) / 255) . \wedge 4) ;$

> subplot(221), imshow( Igris2), subplot(222), imhist( Igris2);

> subplot(223), imshow( Itransformada2 ), subplot(224), imhist

( Itransformada2);
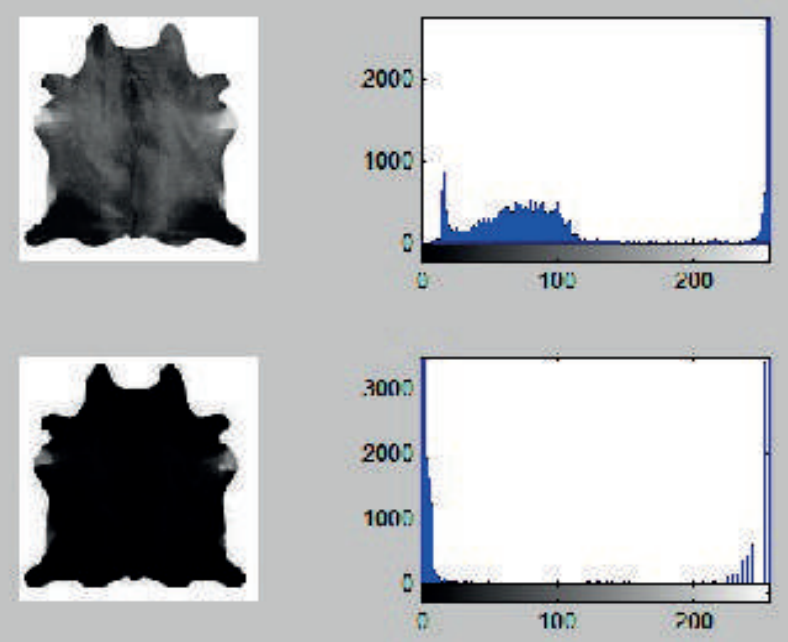

Figura 04. Conversión de imagen de color a tonos de gris.

Como se observa en la columna del lado derecho de la figura 04, el histograma ha sufrido una modificación lo cual facilita la determinación de uno o más umbrales en la zona más apropiada del nuevo histograma. Asimismo, la imagen del lado izquierdo superior se representa de forma más oscura en el lado izquierdo inferior, como producto de la manipulación del histograma.

Con el histograma modificado, se procede a determinar un umbral haciendo uso del método de Otsu tal como fue empleado por Huamaní [03]. Sin embargo, generando un solo umbral y procediendo al proceso de binarización 
se observa que la pieza de cuero no presenta un contorno definido, esto se debe a las zonas claras que aún se encuentran presente en parte del borde de la imagen. Tampoco fue posible tener resultados esperados, cuando se ejecutó una nueva operación de potencia sobre la imagen ya transformada. Por dicha razón, se optó por probar la elección de una umbralización múltiple, lo cual dio mejores resultados. Es así como, se recurrió a comandos propios del Matlab, tal como lo es MULTITHRESH. A continuación, se muestra el procedimiento de uso de dicho comando sobre la imagen transformada "Itransformada2", con la finalidad de tener como retorno tres umbrales por cada imagen transformada.

$>$ umb2 = multithresh (Itransformada2, 3);

$u m b 2=$

\section{6}

$>$ umbral_alto = umb2(3);

Una vez obtenidos los tres umbrales, se procedió a tomar el más alto de todos ellos por ofrecer el mejor resultado de binarización. El mecanismo de binarización se realizó tomando en cuenta el umbral más alto y en función a la ecuación 03.

$$
\begin{gathered}
I_{\text {binarizada }}=\left\{\begin{array}{l}
I_{\text {transformada2 }} \geq \text { umbral }_{\text {alto }}, I_{\text {transformada2 }}=1 \\
I_{\text {transformada2 }}<\text { umbral alto }
\end{array}, I_{\text {transformada2 }}=0\right. \\
>>\text { lbin2 }=\text { Itransformada2 }>=\text { umbral_alto; } \\
>>\text { subplot(121), imshow(Itransformada2) } \\
\text { > > subplot(122), imshow(Ibin2) }
\end{gathered}
$$

Seguidamente, la figura 05 muestra el resultado de aplicar la binarización en una de las imágenes de piezas de cuero vacuno.
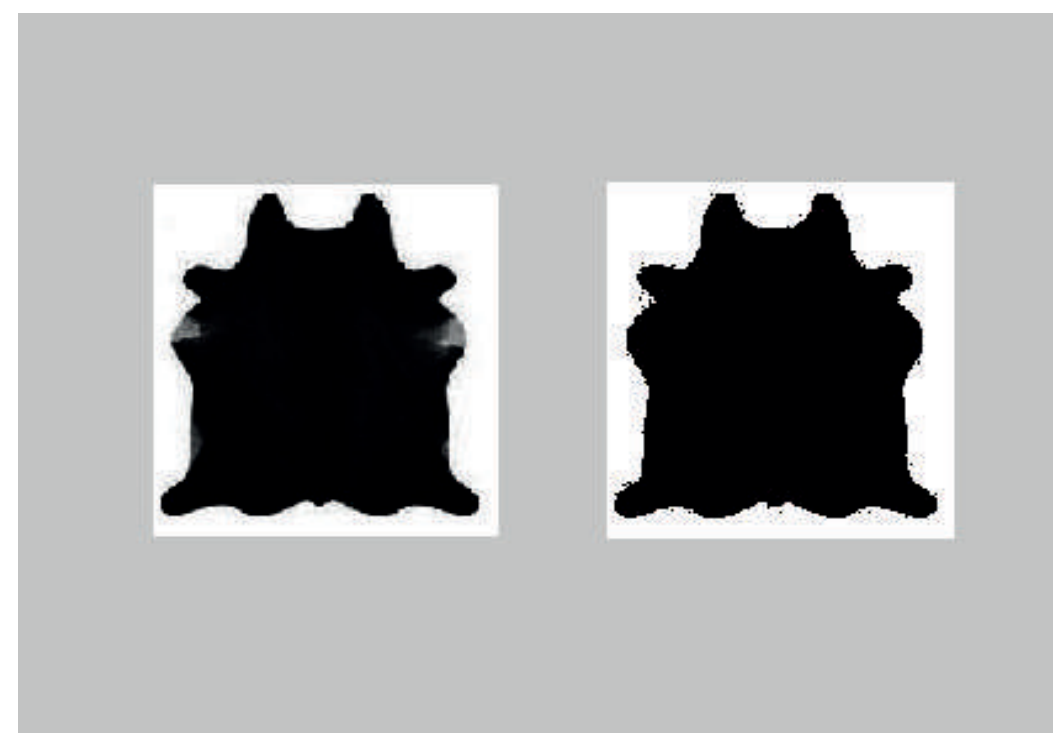

Figura 05. Resultado de la binarización con el umbral más alto. 
Como se aprecia en la figura 05, aún se presenta ciertos pixeles oscuros a través del contorno de la pieza de cuero. Por ello, fue necesario aplicar un proceso de filtrado basado en transformaciones morfológicas para eliminar por completo tal ruido, y así proceder al conteo total de pixeles. No obstante, para aplicar los operadores morfológicos, fue necesario aplicar el negativo con una simple operación de complemento. Esto dio como resultado lo apreciado en la figura 06.

$$
\begin{aligned}
& \text { > > INbin2 = 1- Ibin2; } \\
& \text { > > subplot(121), } \\
& \text { > imshow( Ibin2); } \\
& \text { > > subplot(122), imshow( INbin2); }
\end{aligned}
$$
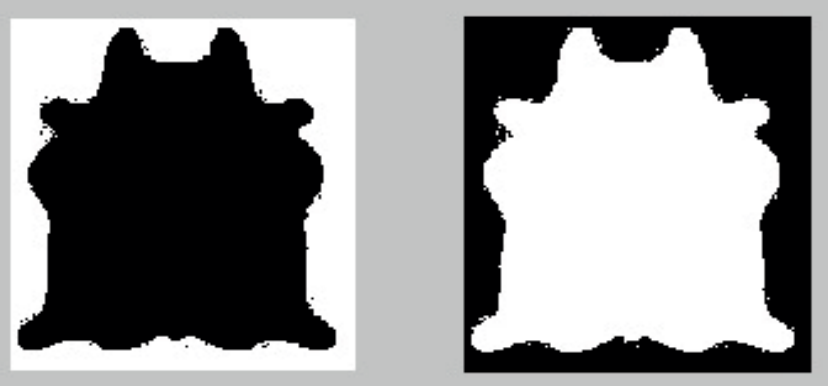

Figura 06. Resultado del negativo de la imagen binarizada.

Una vez realizada la conversión al negativo, se optó por elegir un elemento estructurante de la forma tal como lo muestra la ecuación 04, con la finalidad de que el operador de erosión logre retirar el ruido existente sobre el borde atenuando los pixeles blancos aislados. La forma del elemento estructurante permitió ocuparse de los pixeles vecinos tanto de forma vertical como horizontal.

$$
h[m, n]=\left[\begin{array}{lll}
0 & 1 & 0 \\
1 & 1 & 1 \\
0 & 1 & 0
\end{array}\right]
$$

A continuación, en la figura 07 se representa el resultado de la operación de erosión y particularmente sobre una zona superior izquierda después de haber realizado una operación de zoom. 
$>h=[010 ; 1 \quad 11 ; 010] ;$

$>E E=$ strel('arbitrary', $h)$;

$>$ EINbin2 = imerode (INbin2, EE );

$>$ subplot(121),

$>$ imshow( INbin2),

$>$ axis([ 095095 ])

$>>$ subplot(122),

$>$ imshow( EINbin2),

$>$ axis([ 0950905$])$

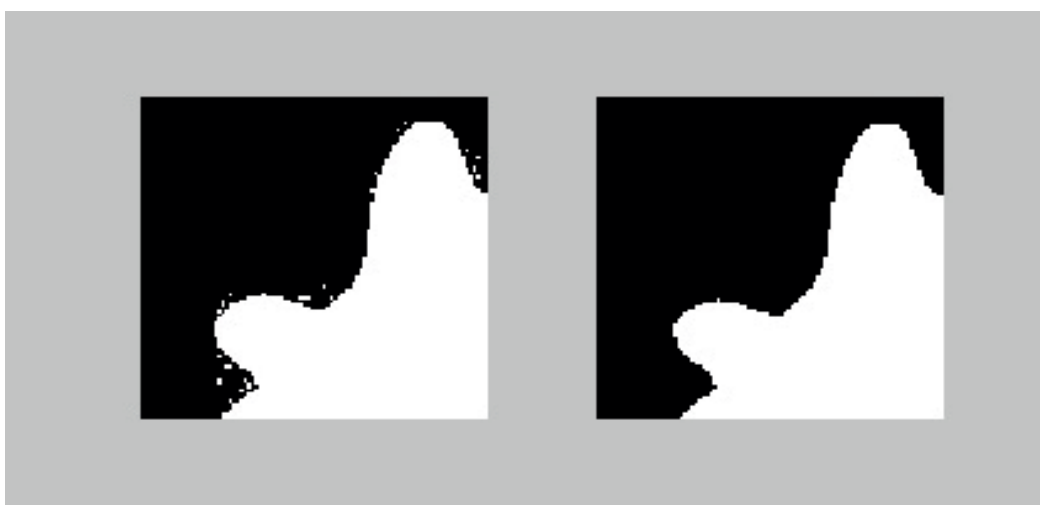

Figura 07. Visualización del resultado del operador morfológico erosión sobre una zona de la pieza de cuero.

Además, en la figura 08 se representa el resultado de toda la técnica planteada sobre las 10 imágenes de muestra que se utilizaron.

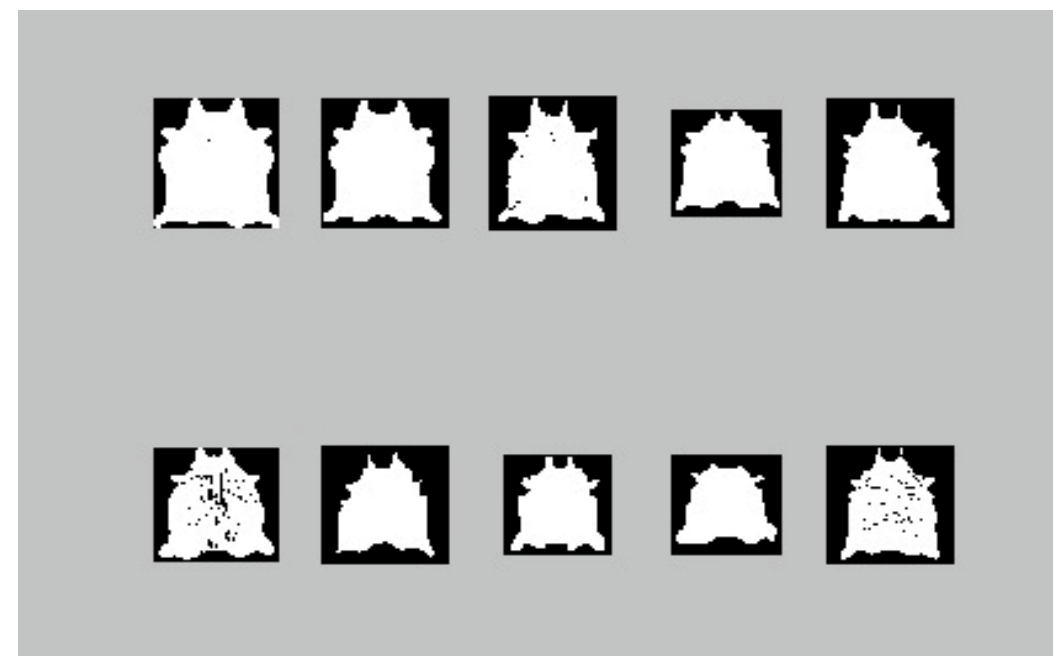

Figura 08. Resultado de la técnica planteada sobre las 10 imágenes de piezas de cuero utilizadas. 
Y, como se puede visualizar, no todas las imágenes de piezas de cuero presentaron uniformidad de pixeles blancos en su interior. Por tal razón, fue necesario aplicar nuevamente un operador morfológico para completar los vacíos, optando esta vez por el operador de cerradura con un elemento estructurante de forma de un cuadrado. Ver la figura 09.

$$
\begin{aligned}
& >>h=\text { ones }(7) ; \\
& >>E E=\text { strel('arbitrary', } h) ; \\
& >>C E I N b i n 2=\operatorname{imclose}(\text { EINbin2 , EE); }
\end{aligned}
$$

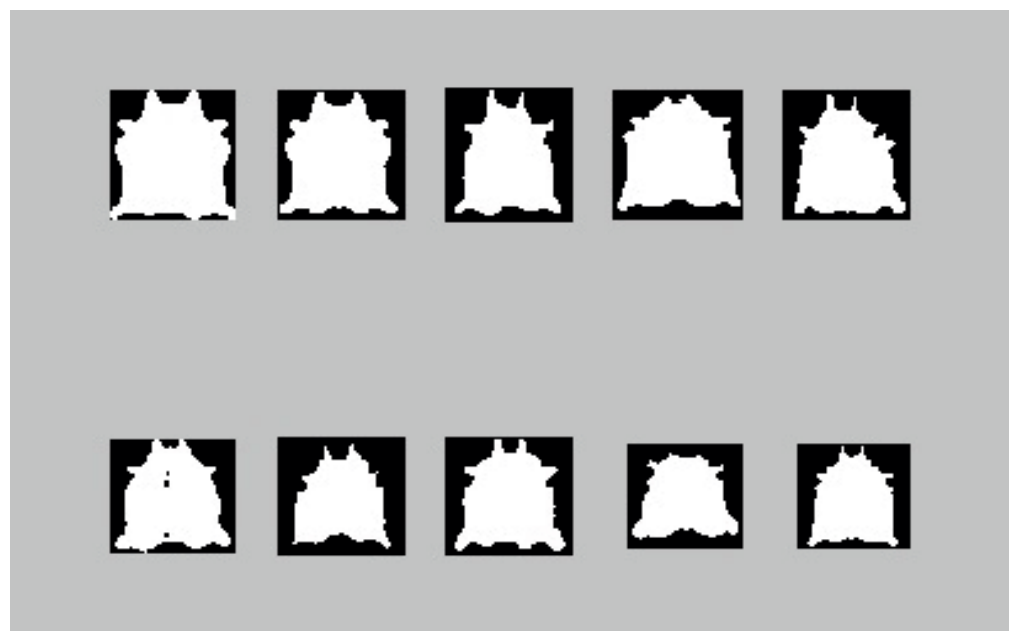

Figura 09. Resultado de la operación morfológica Cerradura, sobre las 10 imágenes de piezas de cuero.

Con este último resultado se puede apreciar que solamente una imagen del grupo de 10 aún presentó ruido (pixeles de color negro), en el interior del contorno. Esto confirma la presencia de variedades de color en la piel del ganado, y obliga a aplicar un nuevo operador morfológico apropiado para rellenar los agujeros.

Si bien es cierto que un operador morfológico del tipo dilatación resolvería el problema, también es necesario aclarar que al aplicar la dilatación la imagen aumenta de tamaño, ocasionando deformación en la pieza de cuero analizada.

Finalmente, se procedió a calcular el total de pixeles blancos en la pieza de cuero realizando una simple operación de suma sobre cada imagen de la figura 09. Ver la ecuación 05.

$$
\text { Total }_{\text {pixeles }}=\sum_{m=1}^{M} \sum_{n=1}^{N} \operatorname{CEINbin}_{2}[m, n]
$$


Donde $\mathrm{M}$ y $\mathrm{N}$ representan el número total de filas y columnas de la Imagen analizada, respectivamente. Además, se observa en la tabla 02 los resultados de la suma de pixeles blancos para cada uno de los archivos utilizados.

>> Total_pixeles2 = sum( $\operatorname{sum}($ MINbin2 $))$;

\section{3) Determinación del área de la pieza de cuero}

Para determinar el área de la pieza de cuero, una vez realizado el conteo de pixeles en el interior de la pieza de cuero segmentada, se debe tener en cuenta muchos parámetros. Entre ellos, la distancia a la cual se ha capturado la imagen digital, así como la resolución del dispositivo de captura. O como también, es suficiente conocer la distancia de captura y el tamaño del pixel en una unidad de longitud, tal como lo señala Zamudio-Lara y otros en su artículo científico [01]. Por lo cual, para conocer tal tamaño de pixel, es necesario contar con una referencia al momento de capturar la fotografía. Esto quiere decir que debemos de tener conocimiento del área de alguna figura referencial en una unidad específica de superficie.

\begin{tabular}{|c|c|c|c|}
\hline & Nombre de Archivo & $\begin{array}{c}\text { Resolución } \\
\text { (pixeles) }\end{array}$ & $\begin{array}{c}\Sigma \Sigma \text { Total_pixeles } \\
\text { (blancos) }\end{array}$ \\
\hline 1 & cuero1.jpg & $457 \times 500$ & 153248 \\
\hline 2 & cuero2.jpg & $233 \times 257$ & 32515 \\
\hline 3 & cuero3.jpg & $209 \times 241$ & 21870 \\
\hline 4 & cuero4.jpg & $217 \times 233$ & 27670 \\
\hline 5 & cuero5.jpg & $217 \times 233$ & 24202 \\
\hline 6 & cuero6.jpg & $229 \times 220$ & 25795 \\
\hline 7 & cuero7.jpg & $225 \times 225$ & 19671 \\
\hline 8 & cuero8.jpg & $225 \times 225$ & 24403 \\
\hline 9 & cuero9.jpg & $375 \times 375$ & 66071 \\
\hline 10 & cuero0.jpg & $325 \times 325$ & 48133 \\
\hline
\end{tabular}

Tabla 02. Tabla con la suma total de pixeles blancos.

Por ejemplo, a continuación, se toma como modelo el siguiente análisis donde se procede a evaluar una fotografía con resolución de $4160 \times 3120$ pixeles que fue capturada a una distancia de 30 centímetros, y en donde se incluyen dos objetos rectangulares, pero solo a uno de ellos se le conoce sus dimensiones reales: de $5 \times 7 \mathrm{~cm}$, lo que equivale a un área de $35 \mathrm{~cm} 2$. Entonces, para determinar el área real del segundo objeto rectangular se procede a determinar la resolución de ambos objetos rectangulares. Es así que, haciendo uso de las técnicas de procesamiento de imágenes, se determina que el primer objeto al cual se le conoce su área tiene una resolución de 
660x560 pixeles; mientras que el segundo objeto presenta una resolución de 1200x880 pixeles. Seguidamente, se procede a utilizar la expresión matemática planteada por Zamudio-Lara y otros [01]. Ver la ecuación 06.

$$
\text { Tamañ } o_{\text {Pixel }}=\frac{A_{c m 2}}{N_{\text {pixel }}}\left[\mathrm{cm}^{2}\right]
$$

Donde, Acm2 representa el área en centímetros cuadrados del objeto de referencia, Npixel corresponde al número de pixeles blancos encontrados en el interior del objeto al cual se desconoce su área, y Tamañopixel hace referencia al área en la unidad de centímetros cuadrados para cada píxel.

De esta forma, tomando en cuenta la ecuación 06 , se procede a reemplazarla con los datos ya conocidos, dando origen al tamaño de pixel en la unidad de centímetros cuadrados.

$$
\text { Tamaño } o_{\text {pixel }}=\frac{35}{660 \times 560}=9.4697 \times 10^{-5} \mathrm{~cm}^{2}
$$

Asimismo, para calcular el área del segundo objeto rectangular en base a su resolución conocida (1200x880 pixeles), se procede a despejar la variable Acm2 de la ecuación 06 dando origen a una nueva ecuación denominada como 07

$$
\begin{aligned}
& A_{c m 2}=\text { Tamaño } o_{\text {pixel }} * N_{\text {pixel }}\left[\mathrm{cm}^{2}\right] \\
& A_{\text {segundo_objeto_en_cm2 }}=\text { Tamaño } o_{\text {pixel }} * N_{\text {pixel }}\left[\mathrm{cm}^{2}\right]
\end{aligned}
$$

$\mathrm{Y}$, reemplazando en la expresión anterior tanto la resolución conocida como el tamaño del pixel en centímetros cuadrados, se tiene:

$$
\begin{aligned}
& A_{\text {segundo_objeto_en_cm } 2}=9.4697 \times 10^{-5} * 1200 * 880\left[\mathrm{~cm}^{2}\right] \\
& A_{\text {segundo_objeto_en_cm } 2}=100 \mathrm{~cm}^{2}
\end{aligned}
$$

En el caso que la distancia de captura de la imagen hubiera sido diferente a 30 centímetros, otra hubiera sido la resolución del segundo objeto y por lo tanto habrá que buscar una proporcionalidad en base a esa nueva distancia. De esta manera, es posible definir una expresión matemática que relacione por proporcionalidad las áreas en función a las distancias, pero como es de esperar, conforme nos acercamos o alejamos en la captura de imágenes pueden surgir detalles como la sombra lo que generaría error en la determinación de la resolución del objeto de interés.

Entonces, teniendo presente que las fotografías de las piezas de cuero fueron obtenidas directamente de distintas páginas web, ignorando la 
distancia a la cual fueron capturadas y desconociendo el área en centímetros cuadrados de cualquier objeto de referencia, se tuvo que asumir el conocimiento de cierto parámetro importante. Fue así como, tomando como referencia la tabla 01, se procedió a suponer el tamaño del pixel en centímetros cuadrados para cada una de las resoluciones de imágenes diferentes, considerando la menor y mayor resolución existente en cada una. Por lo cual, en la tabla 03 se muestra una columna con las suposiciones de tamaño de pixel para cada archivo utilizado, el cual fue elegido con base al contenido del Proyecto de Exportación de Piel Salada a Shanghái, China de L. Sánchez y otros [07], en donde se informa que la empresa Comercializadora e Industrializadora Agropecuaria, CIASA, de México, produce piezas o pieles de cuero con un área aproximada alrededor de $4 \mathrm{~m} 2$ o 43.0556 pies 2 para el ganado bovino resultante de la combinación entre cebú y suizo.

No obstante, dependiendo de la raza del ganado vacuno con el cual se está trabajando, el área de la pieza de cuero variará. Es así como, con el dato supuesto, se procedió a utilizar la ecuación 07 para calcular el área final de la pieza de cuero de interés.

\begin{tabular}{|c|c|c|c|c|}
\hline & $\begin{array}{c}\text { Nombre de } \\
\text { Archivo }\end{array}$ & $\begin{array}{c}\text { Resolución } \\
\text { (pixeles) }\end{array}$ & $\begin{array}{c}\Sigma \Sigma \text { Total_pix- } \\
\text { eles (blan- } \\
\text { cos) }\end{array}$ & $\begin{array}{c}\text { Tamaño Pixel } \\
{\left[\mathbf{c m}^{2} \text { ] }\right.}\end{array}$ \\
\hline 1 & cuero1.jpg & $457 \times 500$ & 153248 & $2.58 \times 10^{-1}$ \\
\hline 2 & cuero2.jpg & $233 \times 257$ & 32515 & $12.34 \times 10^{-1}$ \\
\hline 3 & cuero3.jpg & $209 \times 241$ & 21870 & $18.20 \times 10^{-1}$ \\
\hline 4 & cuero4.jpg & $217 \times 233$ & 27670 & $15.40 \times 10^{-1}$ \\
\hline 5 & cuero5.jpg & $217 \times 233$ & 24202 & $15.40 \times 10^{-1}$ \\
\hline 6 & cuero6.jpg & $229 \times 220$ & 25795 & $15.75 \times 10^{-1}$ \\
\hline 7 & cuero7.jpg & $225 \times 225$ & 19671 & $18.30 \times 10^{-1}$ \\
\hline 8 & cuero8.jpg & $225 \times 225$ & 24403 & $18.30 \times 10^{-1}$ \\
\hline 9 & cuero9.jpg & $375 \times 375$ & 66071 & $6.00 \times 10^{-1}$ \\
\hline 10 & cuero0.jpg & $325 \times 325$ & 48133 & $8.25 \times 10^{-1}$ \\
\hline
\end{tabular}

Tabla 03. Resolución de las fotografías de piezas de cuero con suma total de pixeles y tamaño de pixel supuesto en $\mathrm{cm}^{2}$.

\section{Resultados alcanzados}

Con el grupo de tamaños de pixel supuesto para cada imagen digital utilizada en este artículo y la suma total de pixeles blancos en el área de interés, se procedió a realizar el cálculo del área en la unidad de cm2 utilizando la ecuación matemática 07 ; inmediatamente, con la ecuación 08 se continuó el cálculo para obtener el resultado del área en la unidad de pies2. Se eligió 
la unidad de pies2 debido a que es utilizado comúnmente en el proceso de medición de pieles por parte de las curtiembres.

$$
A R E A_{\text {pies } 2}=\left(A R E A_{c m 2} * 0.00107639\right)\left[\text { pies }^{2}\right]
$$

De esta forma, en la tabla 04, se muestran los resultados del área en pies2, así como también se representa una columna conteniendo el porcentaje de pixeles blancos en relación con la resolución del archivo analizado. Y, tal como se puede observar, el área de la fotografía "cuero7.jpg" es la menor de todas las demás. Así como también, se observa que el porcentaje de pixeles blancos en relación con la resolución total para esa misma figura es el más reducido.

\begin{tabular}{|c|c|c|c|c|}
\hline & $\begin{array}{c}\text { Nombre de } \\
\text { Archivo }\end{array}$ & $\begin{array}{c}\text { Resolución } \\
\text { (pixeles) }\end{array}$ & Área [pies ${ }^{2}$ ] & $\begin{array}{c}\% \text { pixeles } \\
\text { blancos }\end{array}$ \\
\hline 1 & cuero1.jpg & $457 \times 500$ & 42.5583 & 67.0670 \\
\hline 2 & cuero2.jpg & $233 \times 257$ & 43.1885 & 54.2994 \\
\hline 3 & cuero3.jpg & $209 \times 241$ & 42.8440 & 43.4196 \\
\hline 4 & cuero4.jpg & $217 \times 233$ & 45.8669 & 54.7260 \\
\hline 5 & cuero5.jpg & $217 \times 233$ & 40.1182 & 47.8669 \\
\hline 6 & cuero6.jpg & $229 \times 220$ & 43.7306 & 51.2009 \\
\hline 7 & cuero7.jpg & $225 \times 225$ & 38.7478 & 38.8563 \\
\hline 8 & cuero8.jpg & $225 \times 225$ & 48.0689 & 48.2035 \\
\hline 9 & cuero9.jpg & $375 \times 375$ & 42.6709 & 46.9838 \\
\hline 10 & cuero0.jpg & $325 \times 325$ & 42.7432 & 45.5697 \\
\hline
\end{tabular}

Tabla 04. Resolución de las fotografías de piezas de cuero, área en pies $^{2} y$ porcentaje de pixeles blancos.

\section{CONCLUSIONES}

El objetivo principal de este artículo fue determinar el área de una pieza de cuero de ganado vacuno a través de una aplicación básica de visión artificial. Entonces, se concluye que utilizando la combinación de herramientas matemáticas de procesamiento digital de imágenes basadas en manipulación del histograma, determinación del umbral por el método de Otsu y operadores morfológicos de erosión y cerradura, se alcanzó realizar una segmentación del área de interés facilitando así la cuenta de pixeles blancos que permitió calcular la superficie amorfa de la piel o pieza de cuero en la unidad de pies2. Para ello se requirió de la ayuda de un dato supuesto que corresponde al tamaño del pixel en la fotografía capturada y procesada, y el cual fue del tipo ficticio tomando como base el área aproximada señalada 
en el trabajo de L. Sánchez y otros [07]. Por tal razón, es indispensable contar con la referencia del área de cualquier objeto que se encuentre en la escena capturada, y siempre manteniendo la misma distancia al foco del dispositivo de captura. Sin esta información, resultaría dificultoso obtener la estimación del área de alguna región en la unidad de superficie correspondiente.

Asimismo, es importante aclarar que surge cierta dificultad en la segmentación de la zona de interés, cuando la piel del ganado vacuno que se está procesando corresponde a la raza Holstein, debido a que presenta zonas blancas en el contorno de la imagen y muchas veces se confunde con el fondo de la fotografía. Esto obliga a utilizar nuevas técnicas de segmentación basados en análisis de textura, para poder permitir diferenciar uno del otro.

Adicionalmente se afirma que las capturas fotográficas de las pieles de ganado vacuno deben realizarse de forma perpendicular, para evitar la aparición de sombras que perjudicarían el proceso de segmentación. Y, como también, en la toma de fotografías evitar que dichas pieles presenten pliegues porque ello daría una información errónea del área que se desea calcular.

\section{BIBLIOGRAFÍA REFERENCIAL}

A. Zamudio-Lara y otros (2012). "Implementación de un sistema digital para la medición de áreas mediante procesamiento de imágenes utilizando un FPGA", Programación Matemática y Software, vol. 4, No. 1, p. 51-64, ISSN: 2007-3283, [Online]. Disponible: http://www.progmat.uaem.mx:8080/ articulosVol4Num1/vol4num1art6.pdf

J. Báez Rojas y otros (2004). "Segmentación de Imágenes de Color", Revista Mexicana de Física, vol. 50, No. 6, p. 579-587, [Online]. Disponible: http:// www.scielo.org.mx/pdf/rmf/v50n6/v50n6a5.pdf

P. Huamaní (2015). “Umbralización múltiple utilizando el método de Otsu para reconocer la luz roja en semáforos", Revista Scientia, año XVII, No. 17, ISSN 1993-422X, p. 247-262, [Online]. Disponible: http://www.urp.edu.pe/ urp/pdf/scientia17.pdf

A. Alcaim e C. Dos Santos (2011). Fundamentos do Processamento de Sinais de Voz e Imagen. Rio de Janeiro, Brasil: Interciéncia.

G. Pajares y J. De la Cruz (2008). Visión por Computador Imágenes Digitales y Aplicaciones, $2^{\text {a }}$ ed. España: Alfaomega Ra-Ma.

R. Szeliski (2010). "Computer Vision Algorithms and Applications", [Online]. Available: http://szeliski.org/Book/drafts/SzeliskiBook 20100903 draft.pdf

L. Sánchez, N. Pérez y J. Hernández (2018). "Proyecto de Exportación de Piel Salada a Shanghái, China", Universidad Veracruzana, Especialización en Administración del Comercio Exterior, México. Accedido en marzo, 2018. [Online]. Disponible: https://cdigital.uv.mx/ bitstream/123456789/33136/1/hernandezavilajosebernardo.pdf 